\title{
Oportunidades de exportación de la feijoa en el mercado francés
}

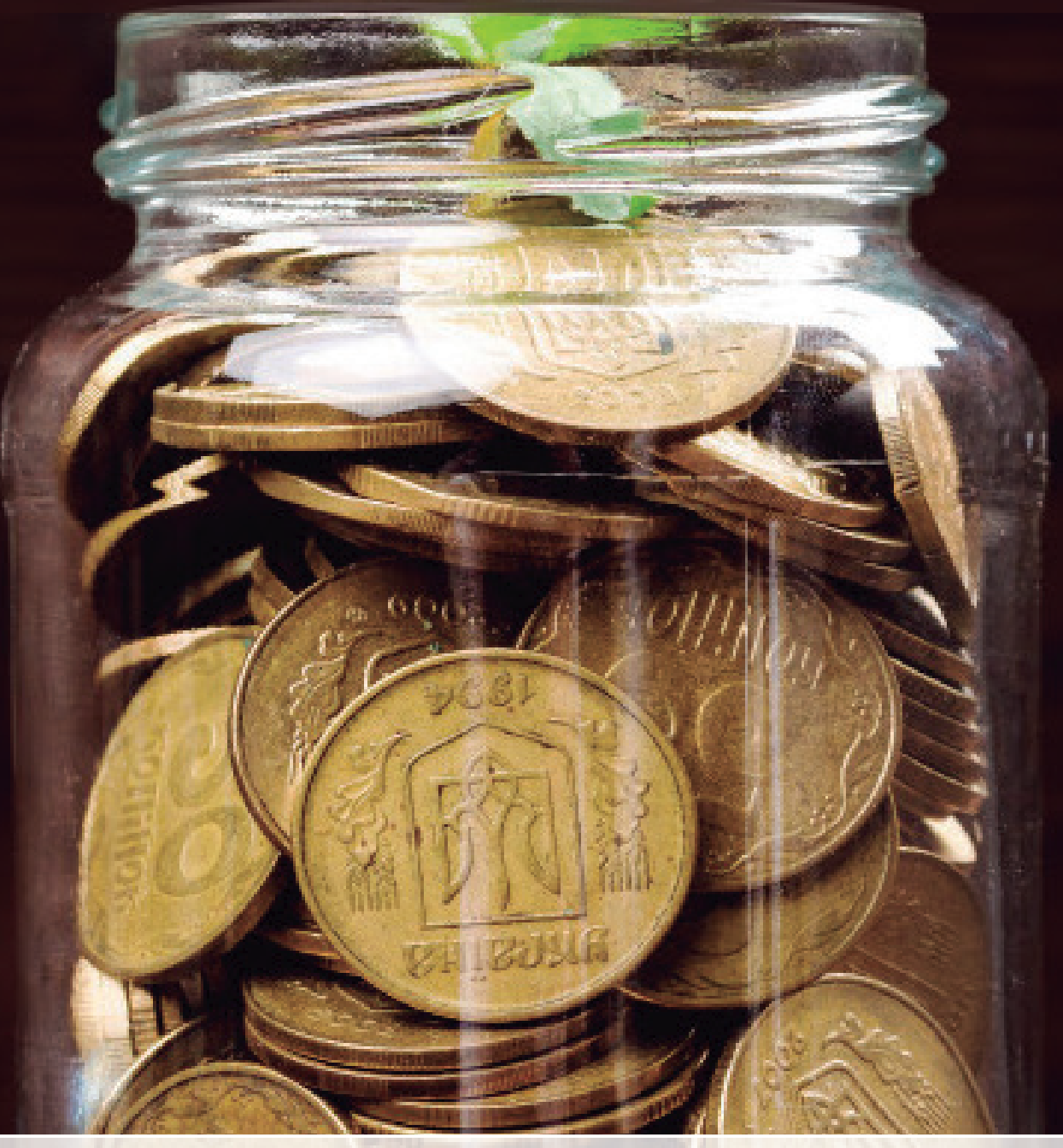

JOHN JAIRO HERNÁNDEZ VILLAMIZAR (10 https://orcid.org/0000-0001-5979-6929 Fundación Universitaria San Mateo / jairohernandezvillamizar@gmail.com

Reconocimiento-SinObraDerivada 4.0 Internacional (CC BY-NC-ND)

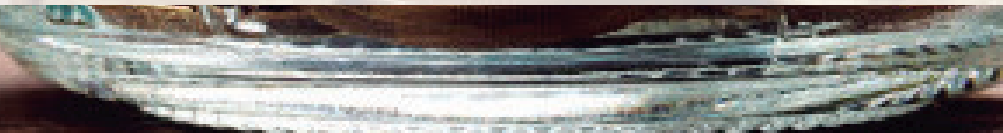


Artículo de investígación

Recepción: 27 de abril de 2020

Aceptación: 04 de agosto de 2020

Cómo citar este artículo

Hernández Villamizar, J. (2020). Oportunidades de exportación de la feijoa en el mercado francés. Revista Colombiana de Ciencias Administrativas

2(1), pp. 26 - 39 Recuperado a partir de: http://cipres.sanmateo.edu.co/

index.php/rcca

\section{Oportunidades de exportación de la feijoa en el mercado francés}

\section{Resumen:}

Este artículo explora las posibilidades de la exportación de la fruta feijoa a Europa, específicamente, a Francia. Este documento pretende analizar al consumidor francés, sus hábitos y sus tendencias en el consumo de fruta y sus derivados; las posibles oportunidades que tiene la fruta en su estado natural y en un desarrollo industrial. La investigación cualitativo-documental sugiere avanzar en un estudio de mercado de productos industrializados con aportes nutricionales para mejorar la introducción de productos derivados de la feijoa al mercado europeo. En la investigación se evidencia cómo los consumidores franceses están a favor de los productos innovadores y con beneficios para su salud sin importar su rango de edad. La feijoa puede llegar a ser una fruta de valor agregado por sus componentes nutricionales; su industrialización aportaría beneficios a los agricultores, exportadores y mercado. Lo anterior adicionaría un portafolio novedoso de nutrientes a la dieta del consumidor francés y concibe un crecimiento al sector agrícola no tradicional.

Palabras clave: exportación; Francia; negocios; hábitos alimentarios; feijoa.

\section{Feijoa export opportunities in the French market}

\section{Abstract:}

This article explores the exporting possibilities for feijoa fruit to Europe specifically to France. This document aims to analyze the French consumer, their habits and their trends in fruit and its derivatives; as well as the possible 
fruit opportunities its natural state, also in industrial development. In this sense, it is based on qualitative-documentary research and suggests to advance in a market study of industrialized products with nutritional inputs to improve the introduction of feijoa-derived products to the European market. Research shows how French consumers are in favor of products innovative and with health benefits, regardless of their age range. The feijoa fruit can become a value-added fruit, given its nutritional components; industrialization would bring benefits to farmers, exporters and the market. It would add a new nutrients portfolio to the French consumer's diet and conceive for growth in the non-traditional agricultural sector.

Keywords: export; France; business; eating habits; feijoa.

\section{Introducción}

La logística de exportación de frutas frescas en Colombia aún se desarrolla bajo parámetros de experiencias. Cañón, Ramírez, Gutiérrez y López (2014), exponen que, existen avances en el desarrollo de modelos matemáticos (como la planeación bajo cuantificaciones de programación lineal), en los trabajos de Caixeta-Filho, Allen, Shustery Saedt, para la industria agroindustrial. Sin embargo, los industriales y empresarios colombianos no aplican estas herramientas de investigación de operaciones que aportarían eficiencia y productividad en sus cadenas de abastecimiento y procesos de exportación.

La feijoa en Colombia es un producto poco comercial, aún es una fruta exótica para nuestro mercado (según Cámara de Comercio de Bogotá CCB, 2011). Por lo tanto, no hay una planeación para la industrialización de sus cadenas de abastecimiento y sus eficiencias productivas. Por lo anterior, las exportaciones se dan por intermediarios que realizan los envíos en cantidades mínimas que no representan unos ingresos significativos a las pequeñas y medianas empresas (Pymes) (CCB, 2010) agrícolas y productoras; en comparación a otros productos agroindustriales como el banano o el café que ya tienen establecidas cadenas de abastecimientos focales alrededor de su producción.

Este documento busca establecer el estado inicial del arte de la feijoa en Colombia; así como sus oportunidades reales de comercialización en Europa, específicamente, Francia porque: 
Se caracteriza por una amplia base de consumidores diversos y sofisticados. Los consumidores franceses son particularmente ávidos (...) y preocupados por los problemas del desarrollo sustentable y el consumo sustentable y el consumo responsable aumenta con fuerza, y Francia dispone hoy de la segunda mayor agricultura orgánica de la Unión Europea (UE). (Portal Santander, 2018).

\section{Fundamentos teóricos}

\section{El consumo de frutas y sus derivados en Francia.}

Los productos saludables están en pleno auge en el mercado europeo. El acceso a servicios de calidad de internet en Europa facilita la búsqueda de información en temas relacionados con el cuidado de la salud, el bienestar de comer sano, los nutrientes de los diversos alimentos (90\% de los hogares dispone de conexión) (Embajada de Francia, 2019). Esto permite que las personas estén informadas sobre sus consumos y los beneficios de los alimentos.

Los consumidores franceses están preocupados por los problemas del desarrollo sustentable; el consumo responsable aumenta con fuerza y Francia dispone hoy de la segunda mayor agricultura orgánica de la UE (Prochile, 2018). Sus esfuerzos se concentran en temas como la calidad con mayor relevancia y lo sustentan basados en sellos, marcas o certificados de calidad de los productos sustentables. Los consumidores prefieren los productos cuidadosamente embalados, creativos y llamativos (Portal Santander, 2018).

Así, "según el estudio de mercados de fruta fresca en Francia en 2011, las frutas más populares consumidas en ese país son: manzanas, bananas, naranjas, mandarinas, clementinas, melones, melocotones, peras, y uvas son las más consumidas con frecuencia" (Ruiz, 2014, p. 16). El mercado francés es exigente al momento de consumir diferentes productos y servicios (Procolombia, 2019); son dados al mercado cultural, al ocio y les gusta testear nuevos productos e innovadores. La alimentación es un rubro fundamental para el mercado francés, prestan la atención al nacionalismo porque los productores nacionales están alineados a sus políticas de sustentabilidad, economía circular. Asimismo, la protección del medio ambiente y el cuidado de la salud; su población está envejeciendo y sus consumos son más conscientes de estas particularidades. 
El consumo de zumo de frutas se mantiene estable entre los consumidores franceses, pese a la incidencia que ha tenido en el mercado la crisis económica que afecta a Europa. Con 1.55 millares de litros consumidos en 2014, los zumos de fruta presentan una estabilidad global de consumo $(-0,6 \%)$ desde el 2009. El consumo anual de zumo en Francia ha progresado en los últimos 25 años; pasó de 2,9 litros por habitante y en 1980, a 23 litros en el 2014 (Oficina económica y comercial de España, 2016). En este sentido, nueve (9) de cada diez (10) franceses consumen jugos de fruta. El $85 \%$ de los jugos se consumen en casa y $80 \%$ durante una comida, es decir, uno (1) de cada dos (2) jugos se consume en el desayuno. Por último, nueve (9) de cada diez (10) jugos consumidos en Francia están comprados (primer criterio: comodidad).

Criterio para hacer jugo en casa: conocer la composición exacta del producto. Francia es el segundo mercado europeo (después de Alemania: 2,4 mil millones de litro). No obstante, el consumo disminuye constantemente desde 2010 (Cougard, 2015). Los franceses tienen una imagen negativa de los productos porque piensan que todos los jugos contienen azúcar. La tasa sobre las bebidas con azúcar (7,5 céntimos por litro) perjudicó a los néctares. Los franceses toman menos desayunos para ganar tiempo; una (1) de cada tres (3) personas no toma desayuno todos los días (momento privilegiado para el consumo de jugos).

Europa es el principal cliente y suministrador de zumos en Francia, encabezando la lista de clientes Bélgica y la de proveedores: Países Bajos. De forma paralela, las adquisiciones de Francia a España son altamente superiores a las expediciones con una tasa de cobertura en el 2015 de un 20\%. En el listado de proveedores, España ha logrado desbancar a Alemania en el segundo lugar del listado de proveedores; alcanza a abarcar un 20\% sobre el total de zumos importados.

En todos los canales de distribución el zumo puro encabeza las ventas (56.2\% en 2014), seguido del zumo a base de concentrado (25.7\%) y los néctares (17.6\%). Las Pymes son los modelos principales para la distribución del zumo de frutas; representan un 69\% de los zumos de fruta vendidos en 2014 con un volumen en baja de 2\% respecto a 2013.

La cuota de mercado de las grandes y medianas superficies (GMS) es siempre largamente superior a la del hard discount (20\%, -2.2 puntos respecto de 2013). Este ve recular sus ventas en volumen en $-9.4 \%$ con respecto a 2013. Por lo que respecta al consumo fuera de casa, los zumos 
de frutas resisten bien; representa un 11\% del volumen vendido (+0.6 \% respecto de 2013) (Navajas, 2016).

Tipos de jugo consumidos en 2016:

- 61\% de pur jus (Jugo Puro): Fruta exprimida.

- 23\% de jus ABC (Jugo a base de concentrado): Jugo reconstituido con agua.

- 6\% de néctar: Entre 25 y 50\% de frutas (jugo, jugo concentrado, purée), con adición de agua y azúcar (Unijus, 2017).

Los sabores favoritos de los franceses en 2016 fueron la naranja (45,9\%), multifrutas (23.5\%), manzana (11.3\%), vitaminados (4.8\%), piña (2.9\%), exóticos (2.8\%), pomelo (2.2\%), uva (1.9\%), frutos rojos $(1.8 \%)$ y albaricoque (0.6\%) (Unijus, 2017). Por otro lado, las tendencias de consumo de bebidas en 2017 de bebidas saludables son las bebidas para completar la alimentación (vitaminas, antioxidantes), súper-frutas, bebidas light y con substituto de azúcar (stevia) (Nutrition santé longévité, 2019).

Los principales targets (población objetivo) de estas bebidas son jóvenes mujeres activas (Agro Media, 2012). La salud y el bienestar tienen mucha importancia en el desarrollo de nuevos jugos y sabores. La innovación y la calidad son factores determinantes para vender jugos en Francia (USDA, 2012).

La tendencia de mercados orgánicos en Francia (2017) es abastecer la demanda de los supermercados que están más dispuestos a vender frutas y verduras orgánicas de orígenes más lejanos. ProNatura (mayorista especializado en la venta de frutas y hortalizas orgánicas) es la primera red de jardineros y arboricultores orgánicos en Francia. Está dispuesto a recibir oferta de frutas orgánicas, sobre todo frutas endémicas, siempre y cuando se respeten las exigencias de la certificación orgánica.

Cabe destacar la importancia que están cobrando los zumos ecológicos (u orgánicos); si bien continúan siendo minoritarios, están progresando a buen ritmo: $+15.1 \%$ en las ventas del 2014 frente a las de 2013. En total, desde 2009 hasta 2014 el crecimiento experimentado por éstos ha sido de un 63\%. Los néctares de frutas también han registrado una progresión considerable (+13.4\%), en tanto que los zumos orgánicos a base de concentrados han visto reducida su cuota de mercado (-49.6\%). 
La tienda especializada Biocoop (primer distribuidor francés de productos orgánicos) se organiza como una red de actores independientes, activistas y comprometidos. Sus pilares son el espíritu de cooperación y el desarrollo de la agricultura orgánica sustentable; la transparencia y la equidad de las relaciones comerciales; la calidad de los productos y la participación del "consumidor actor". En estas tiendas se venden frutas y verduras de su propia red de productores, respetando la temporada.

Además, no se importa fruta en contra estación; sus importaciones provienen principalmente de la UE. Importan frutas exóticas y tropicales que provienen de la agricultura orgánica, del comercio justo y sólo la que sea transportada por barcos para limitar el impacto sobre el medio ambiente (la huella de carbono). Este es el caso de los plátanos y raíces de jengibre del Perú y las piñas de República Dominicana. Para estar presente en este tipo de tienda especializada 100\% orgánica, lo esencial es certificar los productos con agricultura orgánica y/o comercio justo.

\section{Colombia y el cultivo de la feijoa}

Los modelos matemáticos aplicados a la agroindustria en Colombia han explorado las posibilidades de la exportación de feijoa fresca. La preocupación general del sector académico y agroindustrial de Colombia es estudiar alternativas viables de productos fácilmente exportables. Lo anterior, para fortalecer las cadenas de productivas agroindustriales y así tener mayores oportunidades de competir en los mercados globales (Cañón, et al 2014, p. 18).

Los estudios exponen que las características de Colombia a nivel climático le permiten cultivar gran diversidad de frutas. Asimismo, su posición geoestratégica, ambiente de negocios, recursos humanos y su plataforma de exportación la hacen un potencial exportador capaz de competir con otros países latinoamericanos en el mercado de frutas frescas (Cañón et al, 2014).

Figura 1. Árbol de la feijoa.

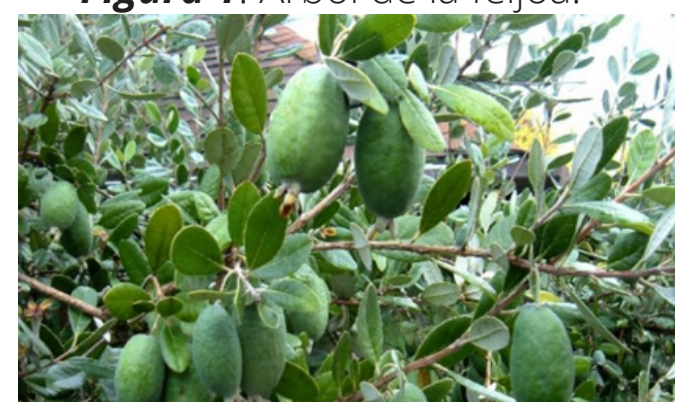

Fuente: MercadoLibre, "Feijoa, árbol de la eterna juventud", s.f. 
El municipio de mayor cultivo de la feijoa en Colombia es Tibasosa (Boyacá), municipio de economía agrícola y sus principales productos son el maíz y la papa. Este municipio produce cerca de 40 toneladas anuales de feijoa que comercializa especialmente en Bogotá como fruta fresca para el consumo. Asimismo, para la exportación como se está registrado en el portal Legicomex de la Tabla 1 y en la Figura 2. Por otro lado, el cultivo de la feijoa genera dos (2) cosechas, la principal en el segundo trimestre del año y la secundaria en el último trimestre, en especial, diciembre y enero.

Tabla 1. Exportaciones de frutas a Europa.

\begin{tabular}{|c|c|c|c|c|c|c|c|c|c|}
\hline Año & Mes & Cantidad(es) & $\begin{array}{c}\text { Peso } \\
\text { en } \\
\text { kilos } \\
\text { netos }\end{array}$ & $\begin{array}{c}\text { Peso } \\
\text { en } \\
\text { kilos } \\
\text { brutos }\end{array}$ & $\begin{array}{l}\text { Valor } \\
\text { FOB } \\
\text { (USD) }\end{array}$ & & $\begin{array}{l}\text { Valor FOB } \\
\text { (COP) }\end{array}$ & $\begin{array}{c}\text { Precio } \\
\text { Unitario } \\
\text { (USD) }\end{array}$ & $\begin{array}{c}\text { Continente } \\
\text { Destino }\end{array}$ \\
\hline & ENERO & 6 & 6 & 19,84 & $\$ 42,60$ & $\$$ & $138.054,24$ & 7,1 & EUROPA \\
\hline & FEBRERO & 130 & 130 & 161,91 & $\$ 489,90$ & $\$$ & $1.619 .609,40$ & 3,8 & EUROPA \\
\hline & JUNIO & 72 & 72 & 88,99 & $\$ 241,20$ & $\$$ & $728.211,74$ & 3,4 & EUROPA \\
\hline & OCTUBRE & 24 & 24 & 30,43 & $\$ 80,40$ & $\$$ & $234.282,38$ & 3,4 & $\overline{\text { EUROPA }}$ \\
\hline & ENERO & 144 & 144 & 205,68 & $\$ 482,40$ & $\$$ & $1.403 .074,87$ & 3,4 & EUROPA \\
\hline & FEBRERO & 32 & 32 & 39,95 & $\$ 195,20$ & $\$$ & $556.706,49$ & 6,1 & EUROPA \\
\hline & FEBRERO & 8 & 8 & 9,52 & $\$ 20,00$ & $\$$ & $57.433,40$ & 2,5 & EUROPA \\
\hline & FEBRERO & 48 & 48 & 58,07 & $\$ 120.00$ & $\$$ & $344.600,40$ & 2,5 & EUROPA \\
\hline & MARZO & 16 & 16 & 22,33 & $\$ 40.00$ & $\$$ & $116.539,20$ & 2,5 & EUROPA \\
\hline & MARZO & 8 & 8 & 9,53 & $\$ 17,71$ & $\$$ & $51.597,73$ & 2,2 & EUROPA \\
\hline & MARZO & 16 & 16 & 21,97 & $\$ 40,00$ & $\$$ & $116.539,20$ & 2,5 & EUROPA \\
\hline & JUNIO & 24 & 24 & 29,68 & $\$ 53,60$ & $\$$ & $161.372,44$ & 2,2 & EUROPA \\
\hline & NOVIEMBRE & 92 & 92 & 111,77 & $\$ 215,15$ & $\$$ & $650.587,78$ & 2,3 & EUROPA \\
\hline & DICIEMBRE & 8 & 8 & 9,85 & $\$ 26,40$ & $\$$ & $79.108,39$ & 3,3 & EUROPA \\
\hline & MARZO & 40 & 40 & 50,31 & $\$ 120,00$ & $\$$ & $333.656,40$ & 3,0 & EUROPA \\
\hline & MARZO & 136 & 136 & 160,67 & $\$ 455,60$ & $\$$ & $1.266 .782,13$ & 3,4 & EUROPA \\
\hline
\end{tabular}

Fuente: Elaboración propia, a partir de Legiscomex (s.f.). 
Figura 2. Exportaciones de frutas a Europa.

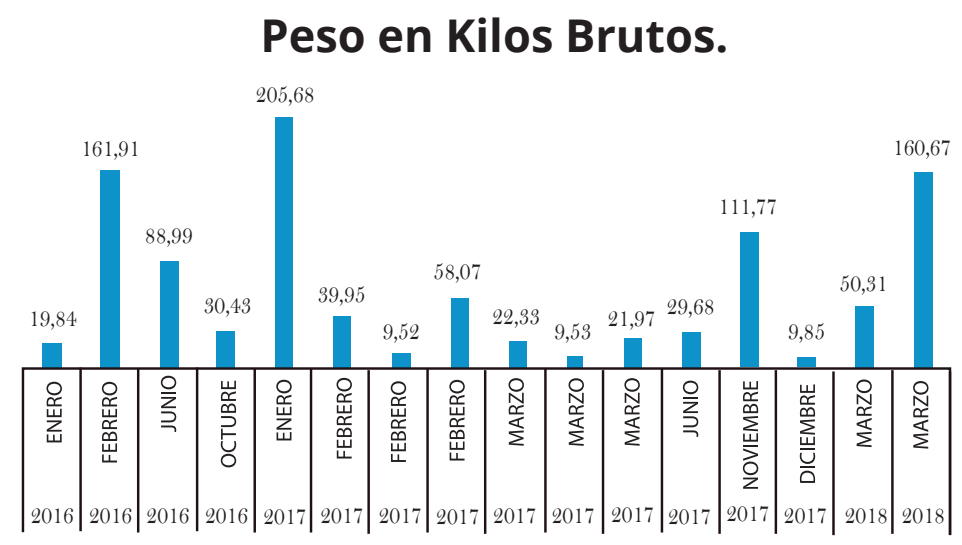

Fuente: Elaboración propia, a partir de Legiscomex (s.f.).

\section{Metodología}

La realización de esta investigación es de tipo cualitativa, a través de la observación externa o no participante. Lo anterior, mediante las técnicas de búsqueda de ventanas de información desde los años 2014 en adelante, principalmente, en bases como ScienceDirect, Researchgate, Scielo, Universidad Nacional y Procolombia. Por otro lado, la investigación se desarrolló en cuatro (4) fases consecutivas relacionadas a continuación:

- Preparatoria: Fase donde se planifican las actividades que se ejecutaran en las fases posteriores, preguntas orientadoras, enfoque y tipos de investigación. Además, se detalló el objetivo de la tutoría-investigación, así como los determinadores del documento final.

- Búsqueda de ventana de información: Consiste en recopilar información necesaria para llegar a una o varias conclusiones o soluciones de la pregunta inicial. Se basa en la búsqueda de matrices de datos y recolección de documentación de expertos en relación con el consumo de frutas en Europa, especialmente, en Francia y el cultivo de feijoa en Colombia. Asimismo, los informes de las perspectivas del mercado francés con respecto a su salud y las tendencias de la población.

- Análisis de la información: Comprueba y valora la información recolectada; evalúa lo hallado; concatena las ideas principales de la documentación y busca la validez de las acciones, datos e información. 
- La última fase fue el desarrollo de conclusiones: Lo anterior, en la medida que los resultados relativos a la eficiencia de la adquisición de los información y documentación nos dan una visión holística del contexto de la feijoa como producto para introducirlo en el mercado europeo.

\section{Resultados}

Las oportunidades de exportar frutas exóticas (con un diferencial de vitaminas como la feijoa con sus valores nutricionales) a regiones como Europa tiene un gran potencial de explotación comercial. Esta fruta contiene:

Agua (85\%), proteínas (0,82\%), carbohidratos (14\%), fibra (3,5\%), ácido ascórbico (28 meq/100 g), ácido cítrico, ácido málico, sacarosa (13\%), glucosa (4,1\%), fructosa (4,6\%). La fruta de la feijoa es rica en vitaminas A, E y $C$ principalmente en la cáscara, presenta altos contenidos de yodo libre (3 mg/100) y minerales mgr/100 gr: potasio $(5,0)$, calcio $(6,0)$, sodio $(4,0)$, magnesio (8,0), fósforo (10,0), hierro 0,50 y bajo contenido de azúcares (Seymour, 1993). Que se emplea cotidianamente en jugos, néctares, helados, yogur, mermeladas, salsa para carne y cocteles. Se reportan características aromáticas que se deben a la presencia de benzoatos de etilo y metilo que representan el 90\% de las sustancias volátiles responsables de la característica resistente de la fruta (Perea, 2014).

La feijoa también ayuda a recuperar las estructuras celulares posponiendo el envejecimiento (Perea, 2014). Otros componentes nutricionales regulan la digestión y tratan algunos problemas de anemia. Además, contiene factor alfa 3, una proteína estudiada que ayuda a microinmunoterapia para frenar la inflamación, la apoptosis y la destrucción articular secundario a la artritis reumatoide (González, Rodríguez y Pariente, 2014).

Para realizar ese desarrollo comercial, primero, se debe estructurar una industrialización del cultivo de la feijoa, en conceptos como cadenas de abastecimiento focal en ecosistemas de simbiosis industrial alrededor de la feijoa. Lo anterior, para obtener Pymes de esta cadena de abastecimiento, produciendo con alto valor agregado no solo fruta en sí, también alimentos comosabajón, dulces, tortas, mermeladas, arequipes, pasas, helados, cremas, panelitas, salsas para carnes, mantecadas, masatos, bocadillos, merengón, incluso vino. Son productos de mayor elaboración y más complejos, con una estrategia de mercado fundamentando en las principales propiedades nutricionales expuesta anteriormente. 


\section{Conclusiones}

Después de realizar la investigación sobre la feijoa y la posibilidad de introducirla en el mercado europeo, específicamente, en Francia; se evidencia que los europeos siempre buscan alimentos que ayuden a su salud y que sean amigables con el medio ambiente.

En la cultura europea se está consumiendo más fruta fresca; están a favor de las economías sostenibles y prefieren consumir las frutas o alimentos cosechados cerca a los centros de consumo (Legoff, 2019), es decir, la idea de llevar el producto colombiano fresco hasta allá (así tuviésemos, o no, la tecnología para preservar los alimentos frescos). En Francia son conscientes de las distancias y las medidas medioambientales; como se identifica en las gráficas de las exportaciones a Europa de la feijoa, lo máximo exportado es el 0.66\% en kilogramos en comparación con las 35 toneladas producidas anualmente en la región de Tibasosa. Por tal razón, entrar en este mercado sería una tarea muy agotadora; requiere una gran inversión de recursos en mercadeo, así como conocimiento del producto por parte de los consumidores.

Sin embargo, en la investigación también se evidenció que los consumidores franceses están a favor de los productos innovadores, con beneficios para su salud. En este sentido, para transportar la feijoa a Francia un cambio de idea es llevarla como un producto con valor agregado, industrializado; haciéndolo exótico, novedoso e interesante para el mercado francés y la región europea.

Por consiguiente, se debe profundizar en el impacto de productos basados en la feijoa mediante estudios de mercados apoyados con entidades del gobierno como Proexport. Aquellos que ayuden a buscar las oportunidades reales que brinda el consumidor europeo para poder generar un marco diferencial hacia las Pymes en las zonas de Colombia que producen esta fruta. Así, se generarían valores empresariales como la co-opetición ${ }^{1}$ donde pequeñas Pymes se unan para exportar mayores volúmenes de productos basados en la feijoa. De esta manera, minimizaría costos de la cadena de suministros y generaría una cadena de abastecimiento focal en los productos de alto valor para el mercado europeo; además, aportaría al aumento de las exportaciones y mejoras en la balanza comercial con productos de mayor valor agregado (Departamento Administrativo Nacional de Estadística, DANE, 2011).

1Se entiende como el acto de cooperación entre empresas competidoras; se dice que las empresas participan tanto en la competencia como en la cooperación. 
La estrategia principal sería la elaboración de productos más avanzados e innovadores, con poder diferenciador y de mayor aceptación en los mercados internacionales. En este sentido, introduciría estratégicamente la competitividad de las Pymes hacia la exportación de productos con alta creatividad, que llamen la atención tanto por los beneficios de la fruta, como en el cumplimiento de las necesidades de este mercado.

\section{Referencias}

Agro Media. (2012). Quelles innovations dans le secteur des boissons sans alcool. Francia: Agro Media.

Cámara de Comercio de Bogotá. (2010). Estudio de mercado sobre las hierbas aromáticas, medicinales y condimentarías en Francia. Bogotá: Editorial CCB.

Cámara de Comercio de Bogotá. (2011). Estudio de mercado sobre la fruta fresca en Francia. Bogotá: Editorial CCB.

Cañón, M., Ramírez, A., Guitérrez-Franco, E., y López-Ospina, H. (2014). Modelo de optimización aplicado a la agroindustria colombiana: Plan logístico integrado para la exportación de feijoa fresca. Boletín de matemáticas, 21(1), pp. 17-31. Recuperado de https://revistas.unal.edu.co/index.php/bolma/ article/view/44156/45447.

Cougard, M. (2015). Le petit monde du jus de fruits tire la sonnette d'alarme. Francia: Les Echos.

Embajada de Francia en Colombia. (2019). Así es Francia [en línea]. Recuperado de https://co.ambafrance.org/-1-Asi-es-Francia-

Departamento Administrativo Nacional de Estadística. (2019). Comercio exterior- exportaciones enero de 2011 [en línea]. Recuperado de https://www.dane.gov.co/files/investigaciones/boletines/exportaciones/ bol_exp_ene11.pdf.

González, D., Rodríguez, A., y Pariente,J. (2014). TNFa-induced apoptosis in human myeloid cell lines HL-60 and K562 is dependent of intracellular ROS generation. Molecular and Cellular Biochemistry, 390, pp. 281-287. 
Legiscomex. (2019). Sistema de Inteligencia Comercial para la gestión y análisis del comercio exterior [en línea]. Recuperado de https://www. legiscomex.com/?SSO-LC-71

Legoff, Y. (2019). Plaisir, praticité et santé restent les valeurs reines. LSA [en línea]. Recuperado de https://www.Isa-conso.fr/plaisir-praticite-etsante-restent-les-valeurs-reines,120951.

Mercado Libre México. (2019). Publicación árbol de feijoa [en línea]. Recuperado de https://articulo.mercadolibre.com.mx/MLM-561937881feijoa-arbol-de- la-eterna-juventud-únicos-JM/

Navajas, P. (2016). El mercado de los zumos en Francia: Estudio bajo la supervisión de la Oficina Económica y Comercial de la Embajada de España en París.

Nutrition santé longévité. (2019). Les grandes tendances en agroalimentaire [en línea]. Recuperado de https://pole-nsl.org/2013/04/08/ les-grandes-tendances-en-agroalimentaire/

Perea, M., Fischer, G., Miranda, D. (2014). Feijoa Acca Sellowiana Berg. En Dallos, M., Matallana, L. y Tirado, A. (Eds.) Biotecnología aplicada al mejoramiento de los cultivos de frutas tropicales (pp. 330-349). Bogotá: Universidad Nacional de Colombia.

Portal Santander Trade Markets. (2019). Analizar mercados, Francia llegar al consumidor [en línea]. Recuperado de https://santandertrade.com/ es/portal/analizar-mercados/francia/llegar-al-consumidor

ProColombia. (2019). Herramientas y servicios para el exportador perfil logístico de exportación a Francia [en línea]. Recuperado de https://www. colombiatrade.com.co/herramientas-del-exportador/perfiles-logisticos-deexportacion-por-pais/perfil-logistico-de-exportacion-francia

Prochile. (2019). Productos Agropecuarios Estudio tendencias productos orgánicos en Francia [en línea]. Recuperado de https://www. prochile.gob.cl/documento-biblioteca/estudio-tendencias-productosorganicos-en-francial 
Ruiz, V. (2014). Formulación de un plan exportador hacia Francia para la microempresa fruta dorada de la ciudad de Manizales. Manizales: Universidad Pontificia Bolivariana.

Unijus. (2017). Les chiffres de la filière. Unijus [en línea]. Recuperado de: http://www.unijus.org/1-/514-economie-marche/532-les-chiffres-de-lafiliere.aspx

USDA. (2012). France non-alcoholic beverage. Francia: USDA. 